\title{
Wound Microbiome, Biofilm Infection and Interactions with Host Immune Cells in Diabetic Foot Ulcers (DFU)
}

Elaine Colomb1, Amitava Das ${ }^{2}$, Suman Santra² and Sashwati Roy ${ }^{2}$

${ }^{1}$ Indiana University School of Medicine; ${ }^{2}$ Indiana University School of Medicine, Department of Surgery, Indiana University Hospital Comprehensive Wound Center (IUHCWC)

\section{Background:}

The treatment of infected diabetic foot wounds places a great burden on our healthcare system. The problem lies in the complexity of treating these wounds, often made more complex by the formation of a bacterial biofilm leading to chronic infection. This critical review discusses current knowledge of biofilm infection and wound healing related to diabetic foot ulcers. Hypothesis: Wound microbiome and biofilm infection subvert immune cell function, specifically the macrophage defense response, which leads to a non-healing chronic wound. In addition to the critical review of literature, a systematic analysis of wound microbiome was performed on fluids obtained from chronic wound patients presented at the IUHCWC.

\section{Methods:}

A comprehensive literature review was performed on DFU microbiome, biofilm infection and interaction with host immune system, and macrophage interactions with biofilm using life sciences and biomedical search engines such as PubMed. Over 50 original and review articles were critically reviewed to derive the information presented in this work. For the wound microbiome analysis, wound fluids from chronic wound patients $(n=20)$ undergoing wound care at IUHCWC were collected under an IRB approved protocol. DNA extraction was performed from the fluid and was subjected to metagenomic 16S rDNA sequencing using a nextgeneration sequencing platform for the microbiome analysis.

\section{Results \& Conclusions:}

The biofilm induces a persistent state of low-grade inflammation via cytokine release and the continuous production of metabolic waste. Up-regulation of genes such as arginine-1 and interleukin-10, as well as a metabolic shift from aerobic glycolysis to oxidative phosphorylation, promote macrophage polarization to an anti-inflammatory, and more importantly, immune suppressive phenotype incapable of clearing the biofilm infection. A detailed analysis of wound fluid microbiome is currently ongoing and will be presented in the poster presentation forum.

Work supported by IMPRS fellowship to EC and NIH R01DK114718 to SR 